\title{
The insignificance of P-R drag in detectable extrasolar planetesimal belts
}

\author{
M. C. Wyatt \\ UK Astronomy Technology Centre, Royal Observatory, Edinburgh EH9 3HJ, UK \\ e-mail: wyatt@roe.ac.uk
}

Received 27 September 2004 / Accepted 13 December 2004

\begin{abstract}
This paper considers a simple model in which dust produced in a planetesimal belt migrates in toward the star due to P-R drag suffering destructive collisions with other dust grains on the way. Assuming the dust is all of the same size, the resulting surface density distribution can be derived analytically and depends only on the parameter $\eta_{0}=5000 \tau_{\text {eff }}\left(r_{0}\right) \sqrt{M_{\star} / r_{0}} / \beta$; this parameter can be determined observationally with the hypothesis that $\beta=0.5$. For massive belts in which $\eta_{0} \gg 1$ dust is confined to the planetesimal belt, while the surface density of more tenuous belts, in which $\eta_{0} \ll 1$, is constant with distance from the star. The emission spectrum of dust from planetesimal belts at different distances from different mass stars shows that the dust belts which have been detected to date should have $\eta_{0} \gg 1$; dust belts with $\eta_{0} \ll 1$ are hard to detect as they are much fainter than the stellar photosphere. This is confirmed for a sample of 37 debris disk candidates for which $\eta_{0}$ was determined to be $>10$. This means that these disks are so massive that mutual collisions prevent dust from reaching the inner regions of these systems and P-R drag can be ignored when studying their dynamics. Models for the formation of structure in debris disks by the trapping of particles into planetary resonances by P-R drag should be reconsidered. However, since collisions do not halt $100 \%$ of the dust, this means that in the absence of planetary companions debris disk systems should be populated by small quantities of hot dust which may be detectable in the mid-IR. Even in disks with $\eta_{0} \ll 1$ the temperature of dust emission is shown to be a reliable tracer of the planetesimal distribution meaning that inner holes in the dust distribution imply a lack of colliding planetesimals in the inner regions.
\end{abstract}

Key words. stars: circumstellar matter - stars: planetary systems: formation

\section{Introduction}

Some $15 \%$ of nearby stars exhibit more infrared emission than that expected from the stellar photosphere alone (e.g., Aumann et al. 1984). This excess emission comes from dust in orbit around the stars and its relatively cold temperature implies that it resides at large distances from the stars, 30-200 AU, something which has been confirmed for the disks which are near enough and bright enough for their dust distribution to be imaged (Holland et al. 1998; Greaves et al. 1998; Telesco et al. 2000; Wyatt et al. 2005). Because the dust would spiral inwards due to Poynting-Robertson (P-R) drag or be destroyed in mutual collisions on timescales which are much shorter than the ages of these stars, the dust is thought to be continually replenished (Backman \& Paresce 1993), probably from collisions between km-sized planetesimals (Wyatt \& Dent 2002). In this way the disks are believed to be the extrasolar equivalents of the Kuiper Belt in the Solar System (Wyatt et al. 2003b).

These debris disks will play a pivotal role in increasing our understanding of the outcome of planet formation. Not only do these disks tell us about the distribution of planetesimals resulting from planet formation processes, but they may also provide indirect evidence of unseen planets in their systems. Models have been presented that show how planets can cause holes at the centre of the disks and clumps in the azimuthal distribution of dust, both of which are commonly observed features of debris disks. Many of these models require the dust to migrate inward due to P-R drag to be valid; e.g., in the model of Roques et al. (1994) the inner hole is caused by a planet which prevents dust from reaching the inner system which would otherwise be rapidly replenished by P-R drag (e.g., Strom et al. 1993), and clumps arise in models when dust migrates inward due to P-R drag and becomes trapped in a planet's resonances (Ozernoy et al. 2000; Wilner et al. 2002; Quillen \& Thorndike 2002). Alternative models exist for the formation of both inner holes and clumps; e.g., in some cases inner holes may be explained by the sublimation of icy grains (Jura et al. 1998) or by the outward migration of dust to the outer edge of a gas disk (Takeuchi \& Artymowicz 2001), and clumps may arise from the destruction of planetesimals which were trapped in resonance with a planet when it migrated out from closer to the star (Wyatt 2003).

The focus of the models on P-R drag is perhaps not surprising, as the dynamical evolution of dust in the solar 
system is undeniably dominated by the influence of P-R drag, since this is the reason the inner solar system is populated with dust (Dermott et al. 1994; Liou \& Zook 1999; Moro-Martín \& Malhotra 2002). However, there is no reason to expect that the physics dominating the structure of extrasolar planetesimal disks should be the same as that in the solar system. In fact the question of whether any grains in a given disk suffer significant P-R drag evolution is simply determined by how dense that disk is (Wyatt et al. 1999). It has been noted by several authors that the collisional lifetime of dust grains in the well studied debris disks is shorter than that of P-R drag (e.g., Backman \& Paresce 1993; Wilner et al. 2002; Dominik \& Decin 2003), a condition which means that P-R drag can be ignored in these systems.

Clearly it is of vital importance to know which physical processes are at play in debris disks to ascertain the true origin of these structures. In this paper I show that P-R drag is not an important physical process in the disks which have been detected to date because collisions occur on much shorter timescales meaning that planetesimals are ground down into dust which is fine enough to be removed by radiation pressure before P-R drag has had a chance to act. In Sect. 2 a simple model is derived for the spatial distribution of dust created in a planetesimal belt. In Sect. 3 this model is used to determine the emission spectrum of these dust disks. A discussion of the influence of P-R drag in detectable and detected debris disks as well as of the implications for how structure in these disks should be modelled and interpreted is given in Sect. 4 .

\section{Balance of collisions and P-R drag}

In this simple model I consider a planetesimal belt at a distance of $r_{0}$ from a star of mass $M_{\star}$ which is producing particles all of the same size, $D$. The orbits of those particles are affected by the interaction of the dust grains with stellar radiation which causes a force which is inversely proportional to the square of distance from the star, and which is commonly defined by the parameter $\beta=F_{\text {rad }} / F_{\text {grav }}$ (Burns et al. 1979; Gustafson 1994). This parameter is a function of particle size and for large particles $\beta \propto 1 / D$. The tangential component of this force is known as Poynting-Robertson drag, or P-R drag. This results in a loss of angular momentum from the particle's orbit which makes it spiral in toward the star. Assuming the particle's orbit was initially circular, the migration rate is:

$\dot{r}_{\mathrm{pr}}=-2 \alpha / r$,

where $\alpha=6.24 \times 10^{-4}\left(M_{\star} / M_{\odot}\right) \beta \mathrm{AU}^{2} \mathrm{yr}^{-1}$ (Wyatt et al. 1999).

On their way in, dust grains may collide with other dust grains. The mean time between such collisions depends on the dust density:

$t_{\text {coll }}(r)=t_{\text {per }}(r) / 4 \pi \tau_{\text {eff }}(r)$,

where $t_{\text {per }}=\sqrt{\left(r / a_{\oplus}\right)^{3}\left(M_{\odot} / M_{\star}\right)}$ is the orbital period at this distance from the star, and $\tau_{\text {eff }}$ is the effective optical depth of the disk, or the surface density of cross-sectional area of the dust (Wyatt et al. 1999). If the collisions are assumed to be destructive then the distribution of dust in the disk can be determined by considering the amount of material entering and leaving an annulus at $r$ of width $\mathrm{d} r$. The steady state solution is that the amount entering the annulus due to P-R drag is equal to that leaving due to P-R drag and that which is lost by collisions (i.e., the continuity equation):

$\mathrm{d}\left[n(r) \dot{r}_{\mathrm{pr}}(r)\right] / \mathrm{d} r=-N^{-}(r)$

where $n(r)$ is the one dimensional number density (number of particles per unit radius), and $N^{-}(r)=n(r) / t_{\text {coll }}(r)$ is the rate of collisional loss of $n(r)$. Since in a thin disk $\tau_{\text {eff }}(r)=$ $0.125 D^{2} n(r) / r$, this continuity equation can be solved analytically to find the variation of effective optical depth with distance from the star (Wyatt 1999):

$\tau_{\text {eff }}(r)=\frac{\tau_{\text {eff }}\left(r_{0}\right)}{1+4 \eta_{0}\left(1-\sqrt{r / r_{0}}\right)}$
$\eta_{0}=5000 \tau_{\text {eff }}\left(r_{0}\right) \sqrt{\left(r_{0} / a_{\oplus}\right)\left(M_{\odot} / M_{\star}\right)} / \beta$

where this distribution has been scaled by the boundary condition that at $r_{0}, \tau_{\text {eff }}=\tau_{\text {eff }}\left(r_{0}\right)$.

This distribution is shown in Fig. 1. The result is that in disks which are very dense, i.e., those for which $\eta_{0} \gg 1$, most of the dust is confined to the region where it is produced. Very little dust in such disks makes it into the inner regions as it is destroyed in mutual collisions before it gets there. In disks which are tenuous, however, i.e., those for which $\eta_{0} \ll 1$, all of the dust makes it to the star without suffering a collision. The consequence is a dust distribution with a constant surface density as expected from P-R drag since this is the solution to $\mathrm{d}\left[n(r) \dot{r}_{\mathrm{pr}}\right]=0$. Dust distributions with $\eta_{0} \approx 1$ have a distribution which reflects the fact that some fraction of the dust migrates in without encountering another dust grain, while other dust grains are destroyed. This can be understood by considering that $\eta_{0}=1$ describes the situation in which the collisional lifetime in the source region given by Eq. (2) equals the time it takes for a dust grain to migrate from the source region to the star, which from Eq. (1) is $t_{\mathrm{pr}}=400\left(M_{\odot} / M_{\star}\right)\left(r_{0} / a_{\oplus}\right)^{2} / \beta$ years.

Figure $1 \mathrm{~b}$ shows the distribution of dust originating in a planetesimal belt $30 \mathrm{AU}$ from a solar mass star for different dust production rates. This illustrates the fact that the density at the centre does not increase when the dust density reduces to a level at which P-R drag becomes important, because even when the disk is very dense a significant number of particles still make it into the inner system. A look at Eqs. (4) and (5) shows that even in the limit of a very dense disk the effective optical depth at the centre of the disk cannot exceed

$\max \left[\tau_{\mathrm{eff}}(r=0)\right]=5 \times 10^{-5} \beta \sqrt{\left(M_{\star} / M_{\odot}\right)\left(a_{\oplus} / r_{0}\right)}$,

which for the belt plotted here means that the density at the centre is at most $4.6 \times 10^{-6}$.

Of course the situation described above is a simplification, since dust is really produced with a range of sizes. Dust of different sizes would have different migration rates, as defined by Eq. (1), but would also have different collisional lifetimes. Equation (2) was derived under the assumption that the dust is most likely to collide with grains of similar size (Wyatt et al. 1999), collisions which were assumed to be destructive. In reality the collisional lifetime depends on particle size, in a way which depends on the size distribution of dust in the disk, and 


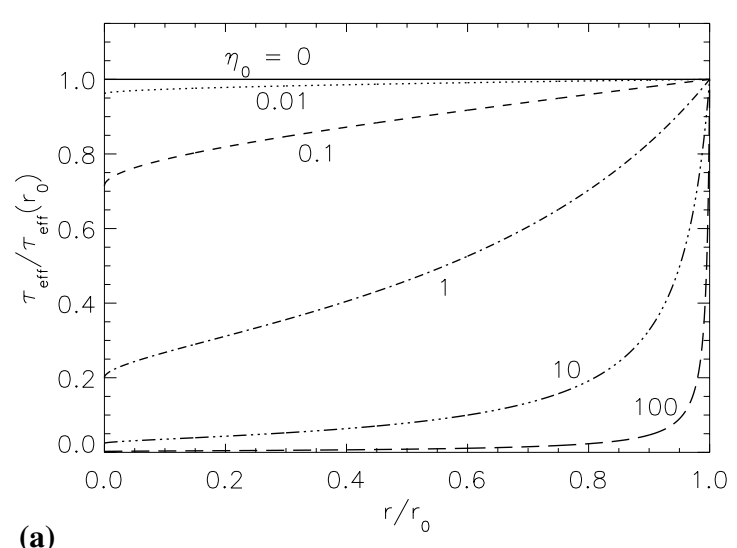

(a)

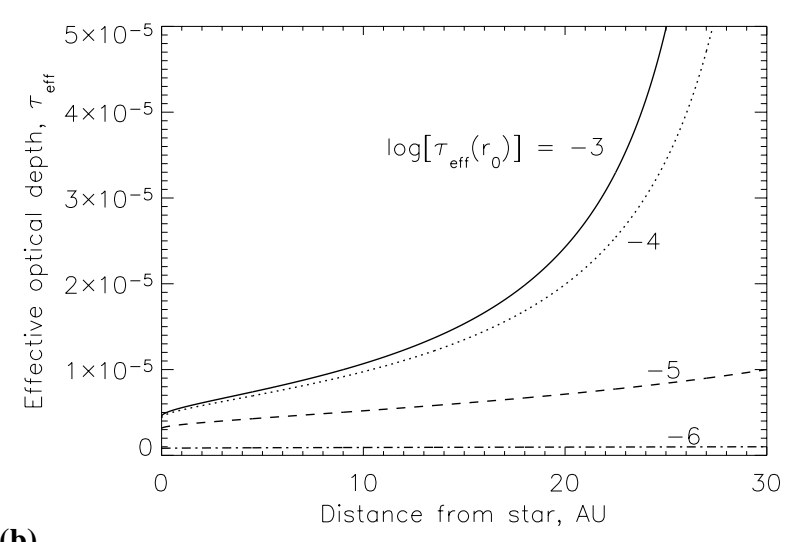

(b)

Fig. 1. The distribution of effective optical depth in a disk resulting from a source of same-sized particles located at $r_{0}$. The particles evolve into the inner disk due to P-R drag and mutual collisions (which are assumed to be destructive). The steady state solution depends on the parameter $\eta_{0}$ which is defined by the source parameters. A value of $\eta_{0}=1$ corresponds to the situation when the collisional lifetime of the source particles (if they suffered no P-R drag evolution) is equal to their P-R drag lifetime. a) shows the functional dependence of the distribution on $\eta_{0}$; b) shows the distribution resulting from a source of particles with $\beta=0.5$ located at 30 AU from a solar mass star, but with different dust production rates that result in different effective optical depths at the source.

the size of impactor required to destroy the particle, rather than result in a non-destructive collision (e.g., Wyatt \& Dent 2002). Once such a size distribution is considered, one must also consider that dust of a given size is not only destroyed in collisions, but also replenished by the destruction of larger particles. The resulting continuity equation can no longer be solved analytically, but must be solved numerically along with an appropriate model for the outcome of collisions between dust grains of different sizes. Such a solution is not attempted in this paper which is more interested in the large scale distribution of material in extrasolar planetesimal belts for which the assumption that the observations are dominated by grains of just one size is a fair first approximation, albeit one which should be explored in more detail in future work.

\section{Emission properties}

For simplicity the emission properties of the disk are derived under the assumption that dust at a given distance from the star is heated to black body temperatures of $T_{\mathrm{bb}}=$ $278.3\left(L_{\star} / L_{\odot}\right)^{1 / 4} / \sqrt{r / a_{\oplus}} \mathrm{K}$. It should be noted, however, that small dust grains tend to emit at temperatures hotter than this because they emit inefficiently at mid- to far-IR wavelengths, and temperatures above black body have been seen in debris disks (e.g., Telesco et al. 2000).

The emission spectrum of dust from planetesimal belts around stars of different spectral type are shown in Fig. 2. The shape of these spectra can be understood qualitatively. At the longest wavelengths all of the dust is emitting in the Rayleigh-Jeans regime leading to a spectrum $F_{v} \propto \lambda^{-2}$. At shorter wavelengths there is a regime in which $F_{v} \propto \lambda$. This emission arises from the dust which is closest to the star. Since dust which has a temperature $\ll 2898 \mu \mathrm{m} / \lambda$ is emitting on the Wien side of the black body curve, this contributes little to the flux at this wavelength. Thus the flux at a given wavelength the comes from a region around the star extending out to a radius $\propto \lambda^{2}$, corresponding to an area $\propto \lambda^{4}$ and so an emission spectrum $F_{v} \propto \lambda$ (see also Jura et al. 1998). For dust belts in which $\eta_{0} \ll 1$ the two regimes blend smoothly into one another at a wavelength corresponding to the peak of black body emission at the distance of $r_{0}$. For more massive disks the shorter wavelength component is much smaller leading to a spectrum which more closely resembles black body emission at the distance of $r_{0}$ plus an additional hot component.

The flux presented in Fig. 2 includes one contentious assumption which is the size of the dust grains used for the parameter $\beta$. The most appropriate number to use is that for the size of grains contributing most to the observed flux from the disk. In general that corresponds to the size at which the cross-sectional area distribution peaks. In a collisional cascade size distribution the cross-sectional area is concentrated in the smallest grains in the distribution. Since dust with $\beta>0.5$ is blown out of the system by radiation pressure as soon as it is created, this implies that $\beta=0.5$ is the most appropriate value to use, which is what was assumed in Fig. 2. However, evolution due to P-R drag has an effect on the size distribution. Since small grains are removed faster than large grains (see Eq. (1)), the resulting cross-sectional area distribution peaks at large sizes (Wyatt et al. 1999; Dermott et al. 2001). Analogy with the zodiacal cloud in which the cross-sectional area distribution peaks at a few hundred $\mu \mathrm{m}$ (Love \& Brownlee 1993) implies that a much lower value of $\beta$ may be more appropriate, perhaps as low as 0.01 for disks in which $\eta_{0} \ll 1$. Thus the fluxes given in Fig. 2 should be regarded as upper limits to the flux expected from these disks (since $\beta>0.5$ regardless). This is particularly true for fluxes at wavelengths longer than $100 \mu \mathrm{m}$, because even in a collisional cascade distribution the emission at sub-mm wavelengths is dominated by grains larger than a few hundred $\mu \mathrm{m}$, since grains smaller than this emit inefficiently at long wavelengths (see e.g., Fig. 5 of Wyatt \& Dent 2002). Inefficient emission at long wavelengths results in a spectrum which is steeper than $F_{v} \propto \lambda^{-2}$ in the Rayleigh-Jeans 


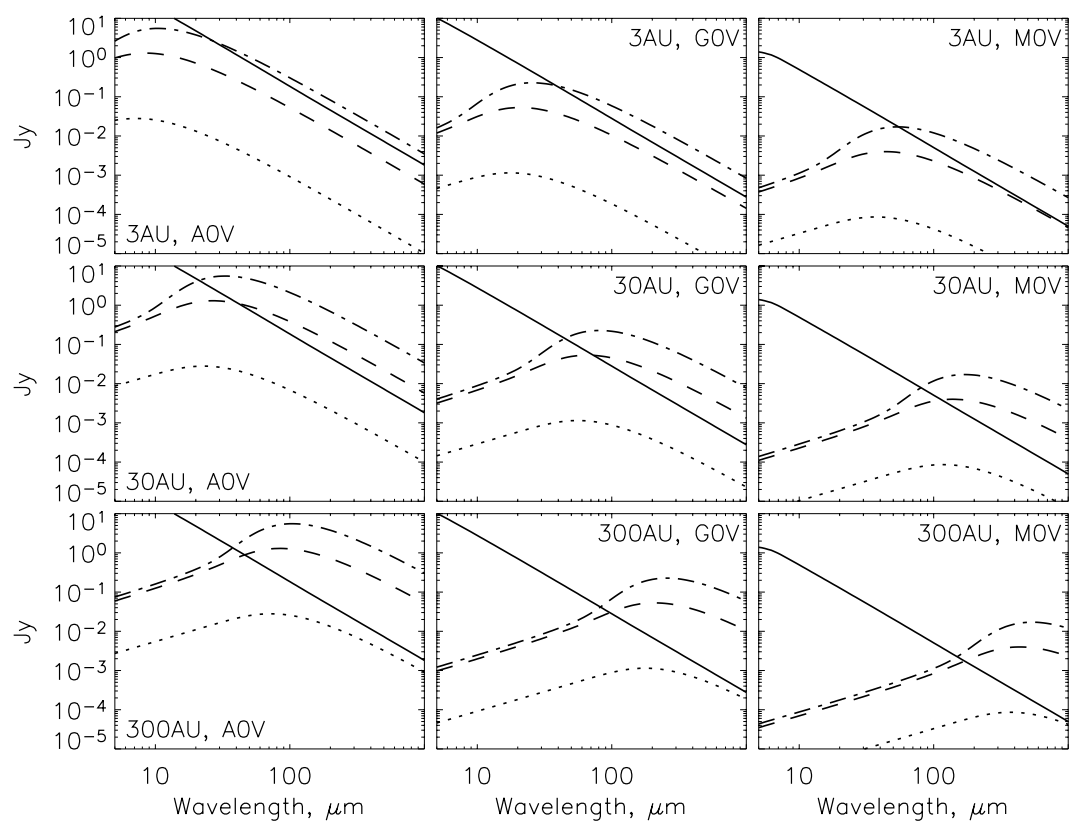

Fig. 2. The spectral energy distribution of emission from dust around main sequence (left) A0, (middle) G0 and (right) M 0 stars. The top, middle and bottom rows of figures show dust from planetesimal belts at 3, 30 and $300 \mathrm{AU}$ respectively. The dotted, dashed and dash-dot lines correspond to dust belts with $\eta_{0}=0.01,1$ and 100 respectively, where this has been converted to an optical depth distribution assuming $\beta=0.5$. In reality the dust may have $\beta<0.5$, so these fluxes should be considered as conservative upper limits to the emission from these disks, even more so because black body emission efficiencies were assumed, whereas typical dust grains have much lower efficiencies at these wavelengths. The fluxes are normalised to stars at a distance of $10 \mathrm{pc}$, but naturally scale with distance squared. The solid line shows the stellar photosphere.

regime. For debris disks the observed spectrum is seen to fall off at a rate closer to $F_{v} \propto \lambda^{-3}$ (Dent et al. 2000).

\section{Discussion}

A disk's detectability is determined by two factors. First is the question of whether the disk is bright enough to be detected in a reasonable integration time for a given instrument. For example, SCUBA observations at $850 \mu \mathrm{m}$ have a limit of a few mJy (Wyatt et al. 2003a) and IRAS observations at 60 and $100 \mu \mathrm{m}$ had $3 \sigma$ sensitivity limits of around 200 and $600 \mathrm{mJy}$. More important at short wavelengths, and for nearby stars, however, is how bright the disk is relative to the stellar photosphere. This is because unless a disk is resolved in imaging, or is particularly bright, its flux is indistinguishable from the stellar photosphere, the flux of which is not generally known with better precision than $\pm 10 \%$. For such cases an appropriate limit for detectability is that the disk flux must be at least 0.3 times that of the photosphere.

The total flux presented in Fig. 2 assumes that the star is at a distance of $10 \mathrm{pc}$. The flux from disks around stars at different distances scales proportionally with the inverse of the distance squared. However, the ratio of disk flux to stellar flux (shown with a solid line in Fig. 2) would remain the same. Given the constraints above, as a first approximation one can consider that the disks which have been detected to date are those with fluxes which lie to the upper right of the photospheric flux in Fig. 2, but with the caveat that such disks can only be detected out to a certain distance which is a function of the instrument's sensitivity.

This allows conclusions to be reached about the balance between collisions and P-R drag in the disks which can have been detected. Fundamentally this is possible because the effective optical depth and $\eta_{0}$ are observable parameters (see next paragraph). The first conclusion is that it is impossible to detect disks with $\eta_{0} \leq 0.01$ because these are too faint with respect to the stellar photosphere. The conclusion about disks with $\eta_{0}=1$ is less clear cut. It would not be possible to detect such disks if they were, like the asteroid belt, at $3 \mathrm{AU}$ from the host stars. At larger distances the disks are more readily detectable. However, detectability is wavelength dependent, with disks around G0V and M0V stars only becoming detectable longward of around $100 \mu \mathrm{m}$, while those around A0V stars are detectable at $>50 \mu \mathrm{m}$. Disks with $\eta_{0} \gg 100$ are readily detectable for all stars, although again there is some dependence on wavelength.

Since most disks known about to date were discovered by IRAS at $60 \mu \mathrm{m}$ this implies that P-R drag is not a dominant factor governing the evolution of these disks, except for perhaps the faintest disks detected around A stars. To check this conclusion a crude estimate for the value of $\eta_{0}$ was made for all disks in the debris disk database (http://www.roe.ac.uk/atc/research/ddd). This database includes all main sequence stars determined in previous surveys of the IRAS catalogues to have infrared emission in excess of that of the stellar photosphere (e.g., Stencel \& Backman 1991; Mannings \& Barlow 1998). To calculate $\eta_{0}$, first only 


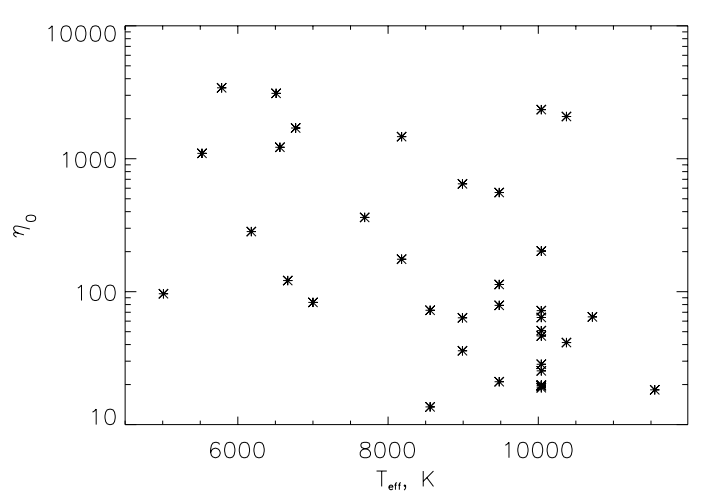

Fig. 3. The value of $\eta_{0}$ for the disks of the 37 stars in the debris disk database with excess flux measurements at two wavelengths plotted against the effective temperature of the stars. The disk around the star HD98800 falls off the plot at $\eta_{0} \approx 10^{5}$.

stars within $100 \mathrm{pc}$ and with detections of excess emission at two IRAS wavelengths were chosen. The fluxes at the longest two of those wavelengths were then used to determine the dust temperature and so its radius by assuming black body emission. Eliminating spectra which implied the emission may be associated with background objects resulted in a list of 37 candidates, including all the well-known debris disks. A disk's effective optical depth was then estimated from its flux at the longest wavelength:

$\tau_{\text {eff }}=F_{v} \Omega_{\text {disk }} / B_{v}(T)$,

where $\Omega_{\text {disk }}$ is the solid angle subtended by the disk if seen face-on, which for a ring-like disk of radius $r$ and width $\mathrm{d} r$ at a distance of $d$ in pc is $6.8 \times 10^{9} \mathrm{~d}^{2} / r \mathrm{~d} r$. The ring width is generally unknown and so for uniformity it was assumed to be $\mathrm{d} r=0.1 r$ for all disks. Finally, $\eta_{0}$ was calculated under the assumption that $\beta=0.5$. All of these stars were found to have $\eta_{0}>10$, with a median value of 80 (see Fig. 3$)^{1}$. All 18 stars (i.e., half the sample) with $\eta_{0}<80$ are of spectral type earlier than $\mathrm{A} 3 \mathrm{~V}$, while stars with disks with $\eta_{0}>80$ are evenly distributed in spectral type. It is worth noting that of the disks which have been resolved, those with ages $\sim 10 \mathrm{Myr}$ all have $\eta_{0}>1000$ ( $\beta$ Pic, HR 4796, HD 141569) while those older than 100 Myr all have $\eta_{0}<100$ (Vega, $\epsilon$ Eridani, Fomalhaut, $\eta$ Corvi).

The fact that the debris disks which have been detected to date have $\eta_{0} \gg 1$ implies that the holes at their centres are not caused by planets which prevent this dust from reaching the inner system. Rather the majority of this dust is ground down in mutual collisions until it is fine enough to be removed by radiation pressure. A similar conclusion was reached by Dominik $\&$ Decin (2003) for debris disks which were detected by ISO. This also means that azimuthal structure in the disks cannot be caused by dust migrating into the resonances of a planet (e.g., Kuchner \& Holman 2003), at least not due to P-R drag

\footnotetext{
1 The biggest uncertainties in the derived values of $\eta_{0}$ are in $r, \mathrm{~d} r$ and $\beta$ : e.g., if black body temperatures underestimate the true radius by a factor of 2 and the width of the ring is $\mathrm{d} r=0.5 r$ then the $\eta_{0}$ values would have to be reduced by a factor of 10 ; changes to $\beta$ would increase $\eta_{0}$.
}

alone. Models of structures in debris disks which have to invoke P-R drag should be reconsidered and would have to include the effect of collisions at the fundamental level to remain viable (e.g., Lecavelier des Etangs et al. 1996), since it appears that P-R drag can effectively be ignored in most detectable disks.

Collisions are not $100 \%$ efficient at stopping dust from reaching the star, and the small amount which does should result in a small mid-IR excess. If no such emission is detected at a level consistent with the $\eta_{0}$ for a given disk, then an obstacle such as a planet could be inferred. However, because of the low level of this emission with respect to the photosphere, it could only be detected in resolved imaging making such observations difficult (e.g., Liu et al. 2004). Even in disks with $\eta_{0} \ll 1$, the resulting emission spectrum still peaks at the temperature of dust in the planetesimal belt itself. This means that the temperature of the dust is a good tracer of the distribution of the planetesimals and a relative dearth of warm dust really indicates a hole in the planetesimal distribution close to the star.

While uncertainties in the simple model presented here preclude hard conclusions been drawn on whether it is possible to detect disks with $\eta_{0} \approx 1$, it is important to remind the reader that fluxes plotted in Fig. 2 used the most optimistic assumptions about the amount of flux emanating from a disk with a given $\eta_{0}$, so that the conclusions may become firmer than this once a proper analysis of the evolution of a disk with a range of particle sizes is done. However, this study does show that detecting such disks would be much easier at longer wavelengths, since photosphere subtraction is less problematic here. Disks which are too cold for IRAS to detect in the far-IR, but which are bright enough to detect in the sub-mm have recently been found (Wyatt et al. 2003a). Thus disks with $\eta_{0} \leq 1$ may turn up in sub-mm surveys of nearby stars. They may also be detected at $160 \mu \mathrm{m}$ by SPITZER (Rieke et al. 2004).

\section{References}

Aumann, H. H., Beichman, C. A., Gillett, F. C., et al. 1984, ApJ, 278, L23

Backman, D. E., \& Paresce, F. 1993, in Protostars and Planets III, ed. E. H. Levy, \& J. I. Lunine (Tucson: Univ. Arizona Press), 1253

Burns, J. A., Lamy, P. L., \& Soter, S. 1979, Icarus, 40, 1

Dent, W. R. F., Walker, H. J., Holland, W. S., \& Greaves, J. S. 2000, MNRAS, 314, 702

Dermott, S. F., Jayaraman, S., Xu, Y. L., Gustafson, B. A. S., \& Liou, J. C. 1994, Nature, 369, 719

Dermott, S. F., Grogan, K., Durda, D. D., et al. 2001, in Interplanetary Dust, ed. E. Grun, B. Å. S. Gustafson, S. F. Dermott, \& H. Fechtig (Heidelberg: Springer-Verlag), 569

Dominik, C., \& Decin, G. 2003, ApJ, 598, 626

Greaves, J. S., Holland, W. S., Moriarty-Schieven, G., et al. 1998, ApJ, 506, L133

Gustafson, B. Å. S. 1994, Annu. Rev. Earth Planet. Sci., 22, 553

Holland, W. S., Greaves, J. S., Zuckerman, B., et al. 1998, Nature, 392, 788

Jura, M., Malkan, M., White, R., et al. 1998, ApJ, 505, 897

Kuchner, M. J., \& Holman, M. J. 2003, ApJ, 588, 1110

Lecavelier des Etangs, A., Scholl, H., Roques, F., Sicardy, B., \& Vidal-Madjar, A. 1996, Icarus, 123, 168

Liou, J.-C., \& Zook, H. A. 1999, AJ, 118, 580 
Liu, W. M., Hinz, P. M., Hoffmann, W. F., et al. 2004, ApJ, 610, L125

Love, S. G., \& Brownlee, D. E. 1993, Science, 262, 550

Mannings, V., \& Barlow, M. J. 1998, ApJ, 497, 330

Moro-Martín, A., \& Malhotra, R. 2002, AJ, 124, 2305

Ozernoy, L. M., Gorkavyi, N. N., Mather, J. C., \& Taidakova, T. A. 2000, ApJ, 537, L147

Quillen, A. C., \& Thorndike, S. 2002, ApJ, 578, L149

Rieke, G. H., Young, E. T., Engelbracht, C. W., et al. 2004, ApJS, 154, 25

Roques, F., Scholl, H., Sicardy, B., \& Smith, B. A. 1994, Icarus, 108, 37

Strom, S. E., Edwards, S., \& Skrutskie, M. F. 1993, in Protostars and Planets III, ed. E. H. Levy, \& J. I. Lunine (Tucson: Univ. Arizona Press), 837

Stencel, R. E., \& Backman, D. E. 1991, ApJS, 75, 905
Takeuchi, T., \& Artymowicz, P. 2001, ApJ, 557, 990

Telesco, C. M., Fisher, R. S., Piña, R. K., et al. 2000, ApJ, 530, 329

Wilner, D. J., Holman, M. J., Kuchner, M. J., \& Ho, P. T. P. 2002, ApJ, $569, \mathrm{~L} 115$

Wyatt, M. C. 1999, Ph.D. Thesis, Univ. Florida

Wyatt, M. C. 2003, ApJ, 598, 1321

Wyatt, M. C., \& Dent, W. R. F. 2002, MNRAS, 334, 589

Wyatt, M. C., Dermott, S. F., Telesco, C. M., et al. 1999, ApJ, 527, 918

Wyatt, M. C., Dent, W. R. F., \& Greaves, J. S. 2003a, MNRAS, 342, 876

Wyatt, M. C., Holland, W. S., Greaves, J. S., \& Dent, W. R. F. 2003b, Earth Moon Planets, 92, 423

Wyatt, M. C., Greaves, J. S., Dent, W. R. F., \& Coulson, I. M. C. 2005, ApJ, in press 